\title{
SIMPLIFICACIÓN ADMINISTRATIVA MUNICIPAL ¿lmposición o iniciativa propia?
}

\author{
Ángela Castillo Mendives \\ Universidad ESAN \\ acastillo@esan.edu.pe
}

\begin{abstract}
Resumen
En el año 2006, gracias a un proyecto de cooperación internacional, se logró la implementación de un proceso de simplificación administrativa en una municipalidad provincial del país para evaluar y modificar la forma como se desarrollaban las fases y los requisitos para la obtención de licencias de funcionamiento. Dado que esta iniciativa fue propiciada por la institución cooperante, el acceso a la información no fue total. Esto generó duplicidad de esfuerzos, una inversión adicional de tiempo, cambios en el presupuesto y, sobre todo, cierta imprecisión en los resultados esperados, lo cual paradójicamente fue observado al finalizar la consultoría por los propios directivos de la municipalidad. Con este caso se demostrará la importancia de considerar los puntos de vista de los miembros de una organización en la toma de decisiones como factor de éxito en cualquier iniciativa de cambio.
\end{abstract}

Palabras clave: simplificación administrativa municipal, cultura organizacional, resistencia al cambio, rediseño de procesos, licencias de funcionamiento, gobiernos locales.

\begin{abstract}
In 2006, thanks to an international cooperation project, an administrative simplification process was implemented in a Peruvian province municipality to evaluate and modify the stages for the issuance of operation permits. As this initiative was organized by the cooperating institution, access to information was not complete and this resulted in overlapped efforts, additional time investment, changes in the budget and, above all, some inaccuracies in the expected results, which were noticed at the end of the consultation process by the municipality's own staff. This case will demonstrate the importance of taking into account the points of view of an organization's members during the decision-making process, as this is the key to success in any change initiative.
\end{abstract}

Key words: municipal administrative simplification, organizational culture, resistance to change, process redesign, operation permits, local governments. 
$E^{2}$ el año 2006, en el marco de un convenio de cooperación internacional, una municipalidad provincial del país fue beneficiada con un proyecto de simplificación de procesos administrativos, específicamente los referidos a la emisión de licencias de funcionamiento.

Había consenso respecto a que ningún país ni empresa podía desarrollarse al margen de la competitividad. Por lo tanto, todos los obstáculos y barreras que se generaran en una organización deberían ser removidos, más aun cuando se tratara de instituciones públicas cuyo desempeño afectaba directamente la eficiencia y la eficacia de las empresas.

De acuerdo con ello, y como primera fase del trabajo, los ejecutores del proyecto invitaron a los alcaldes de diversas municipalidades a participar en esta iniciativa, y la mayoría aceptó de buen grado. Con este consentimiento, el proyecto inició su siguiente fase: la implementación de la propuesta de simplificación. Las coordinaciones continuaron, se realizó el diagnóstico del clima de negocios y se sustentaron sus resultados con investigaciones previas; finalmente, se eligió la municipalidad con la que se llevaría a cabo el proyecto piloto de rediseño de procesos; curiosamente fue la que poseía la mayor población asignada a su jurisdicción. Luego de la firma del convenio, todo quedó listo para la programación de las visitas y el trabajo de campo.

El proyecto de intervención contemplaba la contratación de un consultor encargado de realizar la evaluación de la estructura organizacional y de elaborar la propuesta de simplificación de los procesos considerados principales barreras para un trabajo eficiente en dicha institución. El objetivo principal era reducir los costos y los tiempos invertidos en los trámites realizados en las distintas oficinas e instancias de servicio a la comunidad.

\section{Coordinaciones previas}

Todo estaba listo para comenzar el trabajo; el representante del organismo de cooperación realizó una llamada al alcalde de Buenagracia; el motivo de ella era recordarle el inicio de las visitas a la municipalidad, fechas que estaban señaladas en el cronograma de trabajo.

-Doctor Santisteban, ¿cómo le va? Espero que todo esté dispuesto para el inicio de las visitas de nuestro consultor. ¿Recibió el cronograma que le envié por la mañana?

-Sí, lo recibí, y como le comentaba en la reunión del viernes, mi personal está preparado para empezar el trabajo de inmediato. Pero dígame, ¿cuántas oficinas requieren? ¿Necesitan utilizar algún material especial? Por favor, no dude en solicitar todo lo que le parezca conveniente.

-Pierda cuidado, lo importante es que a nuestro personal se le otorgue todas las facilidades para la recolección de la información. En cuanto al ambiente de trabajo, es justamente el mismo utilizado para cada proceso; como comprenderá, lo que necesitamos es observar cómo se realizan, así que no se preocupe por darnos un ambiente especial.

-Perfecto. Entonces, lo único que le agradecería es que me indique en un documento simple los datos de las personas que estarán trabajando con nosotros en la municipalidad. Cualquier cosa, ya sabe, pueden llamarme al celular o buscarme en mi despacho. 
-Muchas gracias, doctor. Le enviaré un e-mail para confirmarle los datos, y además le reenviaré nuestro cronograma para que pueda señalar la etapa en la que nos encontramos.

Luego de ello, el representante del organismo de cooperación envió el e-mail con los datos solicitados para que la visita del consultor fuera exitosa, sobre todo teniendo en cuenta que esta se realizaría la mañana siguiente.

Antes de salir a una reunión, le pidió a su asistente que llamara a la municipalidad para confirmar dicha cita. Por la tarde, el asistente del proyecto hizo la llamada al despacho de alcaldía, pero sólo pudo comunicarse con la secretaria del alcalde, quien le indicó lo siguiente:

-Señor, lamentablemente no cuento con la información que me indica, y en este momento es imposible comunicarse con el doctor, ya que acaba de salir a un evento que le va a tomar toda la tarde.

-Pero verá, las coordinaciones ya están hechas, el motivo de mi llamada solo es confirmar la visita de nuestro consultor para el día de mañana.

-Mire, la única opción es que usted me indique algún documento con el que yo pueda corroborar lo que me está diciendo. De lo contrario, no podré ayudarlo.

-Como le digo, por la mañana hemos enviado un e-mail al alcalde en el cual le hicimos llegar toda la información. ¿Podría por favor verificarlo?

-En ese caso, ustedes debieron haberme copiado el e-mail, sin ello no tengo cómo acceder a la correspondencia del doctor.
Aunque, claro, puede hacerme llegar un documento y yo podría acomodar una cita en la agenda.

-Muy bien, en este momento le haré llegar un documento por fax. Entonces, señorita, ¿con esto ya no habría ningún problema, verdad?

-¿Perdón, señor? Creo que usted se está equivocando, cuando le digo que me envíe una carta es para que su solicitud sea procesada con las demás. Le recuerdo que esto no puede hacerse por fax, sino por mesa de partes, pero ya tendría que ser mañana, porque en media hora termina la atención. Una vez que la solicitud llegue a mi oficina, lo conversaría con el doctor Santisteban, y si él está disponible, procederé a responderle, por eso no debe olvidar poner una fecha $y$ hora... Aunque para ayudarlo con eso, le sugiero que considere las fechas a partir de los próximos quince días siguientes; antes realmente lo veo imposible.

El asistente estuvo tentado de colgar el teléfono, pero se abstuvo y agradeció a la secretaria; de inmediato procedió a llamar al coordinador del proyecto, quien tuvo que abandonar su reunión y retornar a su oficina para arreglar el malentendido.

Finalmente, se pudo realizar la visita programada, pero una semana después de la fecha prevista en el cronograma.

\section{Primera visita a la Municipalidad de Buenagracia}

El consultor Pedro Calderón fue la persona designada para implementar el proceso de simplificación administrativa en la Municipalidad Provincial de Buenagracia, institución con un complejo y arraigado 
sistema administrativo, donde tuvo que poner en juego todas sus estrategias de empatía con el propósito de obtener la información necesaria.

El día que inició sus visitas a la municipalidad, Pedro Calderón se dirigió a la oficina del gerente municipal, doctor Juan Trillo, con quien había entablado contacto desde las reuniones de planificación del trabajo y era la contraparte designada por el alcalde para este proyecto. El doctor Trillo debía proporcionarle las facilidades necesarias para realizar el levantamiento de información.

-Buenos días, señorita. Soy el consultor Pedro Calderón. Tengo una cita con el doctor Trillo.

-Buenos días, tome asiento, por favor.

La secretaria ingresó a la oficina de su jefe y Pedro alcanzó a oír a un desentendido doctor Trillo tratando de recordar la cita con el consultor; finalmente la secretaria le dio el siguiente mensaje:

-El doctor Trillo le pide que lo disculpe, señor Calderón; en este momento no puede atenderlo, pero me ha encargado a mí que lo oriente. ¿En qué consiste el trabajo que va a realizar aquí?

-Soy el consultor designado para ejecutar el proyecto de simplificación municipal. A partir de hoy empezamos las visitas para evaluar los procedimientos seguidos en la entrega de licencias de funcionamiento...

A medida que Calderón iba explicando esto, la secretaria cambiaba su cordialidad por una actitud de desconfianza.
-Así que usted es quien va a supervisar a los de Desarrollo Económico... Bueno, le enseño las oficinas, tiene que subir las escaleras, al final del pasillo encontrará una sala grande con una larga cola de gente esperando, allí debe preguntar por el licenciado Torres, gerente de Desarrollo Económico Local. Cualquier cosa, estaré en el primer piso.

-¡Gracias! -le dijo Calderón a la secretaria y siguió su camino pensando en la información que debía recolectar.

Cuando Calderón llegó a la gerencia de Desarrollo Económico Local, le costó mucho trabajo llegar hasta una de las ventanillas, y pensó que la secretaria no habría podido describir mejor la situación que estaba viendo.

-Disculpe, mi nombre es Pedro Calderón, busco al licenciado Torres, podría...

-iSeñor, si quiere ser atendido tiene que hacer su cola! El siguiente, por favor -interrumpió una malhumorada empleada de ventanilla a quien apenas alcanzaba a distinguir por la cantidad de papeles que tenía frente a ella.

-Disculpe -repitió Pedro- soy el consultor del proyecto...

No terminó de mencionar estas palabras cuando las personas que esperaban empezaron a silbar y gritar -casi en coro- que todos estaban apurados y que debía hacer cola como todos ellos.

Incómodo, Pedro decidió salir de la sala e hizo algunas llamadas desde su celular. Al cabo de media hora, salió una tímida secretaria que -casi en secreto- lo dirigió por otra puerta de ingreso a las oficinas 
de la gerencia, mientras le explicaba que hubiera sido mejor que los llamara primero: «Bueno, así es la gente, siempre lo mismo».

-Si usted viniera todos los días, ya estaría acostumbrado -le dijo con mucha resignación, mientras Pedro seguía abrumado por la situación en la que había estado.

-Espere un momento, lo voy a anunciar con el gerente -agregó, y luego de un momento volvió y lo hizo pasar.

-¿Cómo le va, señor Calderón?, disculpe la demora, he recibido una llamada del gerente municipal y me dice que hoy empieza el trabajo -le dijo el licenciado Torres-. Tome asiento, por favor, ¿desea algo de beber? Martha, ¿nos puedes traer dos gaseosas?

Calderón empezó a explicarle al gerente los motivos de su visita y le mostró lo que había planificado para ese día, según el cronograma del proyecto. Le indicó que el primer paso era realizar un diagnóstico de los diversos procesos administrativos relacionados con la entrega de licencias en la municipalidad.

Mientras tanto, el gerente compartía su atención con otras actividades, la revisión de sus e-mails y las llamadas de su celular. A Pedro Calderón le parecía que, por momentos, el gerente pensaba en cualquier cosa, menos en lo que él le planteaba.

-Bueno, todo está muy bien, estoy de acuerdo con todo; pero verá, hay un pequeño detalle: muchas de las cosas de las que usted me está hablando no las hacemos aquí, sino que dependemos de otras áreas, y allí sí que no voy a poder ayudarlo, pero si desea en este momento le hago «el link» con los encargados.

Sin dejarlo responder, se levantó de su silla y salió a pedirle a la secretaria que acompañara al consultor, pero al no verla regresó sobre sus pasos.

-Ah, me olvidaba, aprovecho para entregarle la hojita informativa que tenemos en la gerencia, aquí va a encontrar todos los requisitos que debe cumplir la gente que viene a pedir su licencia. Como verá, es muy simple. ¿Sabe?, nos ha costado mucho llegar al orden y la rapidez con que trabajamos en la actualidad. Mi secretaria lo acompañará apenas regrese.

Pedro Calderón sintió que lo estaban despachando, así que no hizo mayor comentario. Se levantó, se despidió con un apretón de manos y esperó junto al escritorio de la secretaria mientras revisaba la hojita. En eso vio venir apresurada a Martha con las gaseosas que su jefe le había solicitado.

-¡Señor Calderón!, ¿ya terminó su reunión?

-Sí. Por favor, ¿podría indicarme dónde queda la Gerencia de Desarrollo Urbano? Y dígame, ¿hay aquí una oficina de entrega del certificado de Defensa Civil?

-Bueno, la verdad no, pero como ese es un requisito, la gente tiene que ir a la Av. Nogales. Ahí está Defensa Civil. Venga por acá, por favor.

Entretanto, lo conducía a la Gerencia de Desarrollo Urbano, en la cual ya lo esperaba una señorita que Martha señaló como la jefa de Catastro. Luego de la presentación de rigor, el consultor sostuvo una breve 
conversación con esta persona sobre los procesos que se realizaban en esa oficina y en el resto de la gerencia. Sin embargo, no quedó muy satisfecho porque había pensado que lo atendería el gerente del área.

Al salir de la oficina, Pedro consideró que para ser primer día ya había tenido suficiente, así que abandonó la municipalidad.

\section{Trabajo en marcha}

Pedro iba continuamente a la municipalidad. Lo curioso fue que, luego de su complicada primera visita, los empleados se mostraban preparados a atenderlo e incluso varios de ellos le comentaron que, debido a su presencia, habían tenido que volver a usar los uniformes que alguna vez el alcalde había mandado a diseñar para ceremonias y ocasiones especiales.

Como le había planteado al alcalde cuando se entrevistó con él, creía que lo más conveniente era revisar su borrador del diagnóstico del proceso de otorgamiento de licencias con los propios empleados de las áreas encargadas, con la finalidad de involucrarlos en el análisis, motivarlos a dar su opinión y, quizá, a proponer iniciativas de cambio interesantes. Su experiencia le había enseñado que los cambios se introducen con mayor éxito cuando mayor es la participación de los miembros de la organización en el proceso.

El cronograma de trabajo había tenido que modificarse varias veces por la inexistencia de documentos o la dificultad para acceder a ellos; pero por fin, transcurridas varias semanas, Pedro se sentía listo para presentar un informe preliminar respecto al desarrollo de las actividades en la Municipalidad de Buenagracia.

En todo momento, el consultor trató de ser objetivo en sus apreciaciones frente a los procesos que había observado y analizado. Al terminar de elaborar su informe llegó a la conclusión de que en esta municipalidad ya no era necesario esperar más; la situación era tal que, aun sin contar con toda la información requerida, ya era posible pasar a la siguiente etapa: la implementación de la reforma de los procesos. De inmediato, procedió a preparar su propuesta de intervención.

Al día siguiente, acudió muy temprano a la municipalidad para reunirse con los directivos, a quienes debía presentar los avances de su trabajo, en la medida en que ellos eran, finalmente, los decisores de dicha intervención y, además, los principales interesados en las potenciales mejoras que les propondría.

Durante su exposición, describió cada fase y la contrastó con su propuesta de modificación. Seguro de la viabilidad de sus planteamientos, argumentó el rediseño de acuerdo con el cambio que él consideraba inminente en la institución.

- ¡Y finalmente, señores, estos son los principales «cuellos de botella», los cuales eliminaríamos con nuestra propuesta de intervención! Como verán, poseemos el respaldo suficiente para ejecutarla de inmediato-Pedro concluía entusiasmado.

Mientras pronunciaba las últimas palabras, el alcalde aún observaba el diagrama de flujos algo escéptico, y el gerente municipal pensaba en restablecer antiguos contactos. 


\section{Preguntas para la discusión}

- ¿Cree usted que el alcalde de Buenagracia comunicó a los empleados de la municipalidad la visita de Pedro Calderón y el proyecto en general? ¿Cómo cree usted que haya tomado el personal el trabajo del consultor?

- ¿Considera usted que la participación del personal influiría en los resultados del proyecto? Explique en qué medida.
- ¿Cree usted que sería pertinente que en una posterior reunión también participaran los subordinados de cada gerencia?

- ¿Cuál cree que sea la respuesta del alcalde y los gerentes presentes en la reunión acerca de los inminentes cambios?

- ¿Considera que el proyecto de simplificación administrativa será viable en una institución como Buenagracia? 